University of Nebraska - Lincoln

DigitalCommons@University of Nebraska - Lincoln

Faculty Publications from Nebraska Center for Materials and Nanoscience

Materials and Nanoscience, Nebraska Center for (NCMN)

June 2003

\title{
Scattering of elastic waves in damaged media
}

Liyong Yang

University of Nebraska - Lincoln, lyang4@unl.edu

Joseph A. Turner

University of Nebraska - Lincoln, jaturner@unl.edu

Follow this and additional works at: https://digitalcommons.unl.edu/cmrafacpub

Part of the Nanoscience and Nanotechnology Commons

Yang, Liyong and Turner, Joseph A., "Scattering of elastic waves in damaged media" (2003). Faculty Publications from Nebraska Center for Materials and Nanoscience. 67.

https://digitalcommons.unl.edu/cmrafacpub/67

This Article is brought to you for free and open access by the Materials and Nanoscience, Nebraska Center for (NCMN) at DigitalCommons@University of Nebraska - Lincoln. It has been accepted for inclusion in Faculty Publications from Nebraska Center for Materials and Nanoscience by an authorized administrator of DigitalCommons@University of Nebraska - Lincoln. 


\title{
Scattering of elastic waves in damaged media
}

\author{
Liyong Yang and Joseph A. Turner ${ }^{\mathrm{a})}$ \\ Department of Engineering Mechanics, W317.4 Nebraska Hall, University of Nebraska-Lincoln, Lincoln, \\ Nebraska 68588-0526
}

(Received 31 May 2002; revised 17 February 2003; accepted 26 February 2003)

\begin{abstract}
The scattering of elastic waves in a medium with damage from microcracking is discussed. The influence of damage from penny-shaped microcracks within a homogeneous medium is considered. The microcracks are assumed to be randomly oriented and uniformly distributed. Explicit expressions are derived for the attenuation of longitudinal and shear elastic waves in terms of the damage parameter and the effective elastic moduli of the medium. A generalized tensor-based approach is used such that the results are coordinate free. The derivation is based upon diagrammatic methods. The problem is formulated in terms of the Dyson equation, which is solved for the mean field response within the limits of the first-order smoothing approximation. The longitudinal and shear attenuations are discussed in terms of their frequency dependence and damage dependence. In particular, the attenuations are shown to scale linearly with the damage parameter. (C) 2003 Acoustical Society of America. [DOI: 10.1121/1.1570444]
\end{abstract}

PACS numbers: 43.20.Bi, 43.20.Gp, 43.35.Cg [DEC]

\section{INTRODUCTION}

The scattering of elastic waves in complex media, particularly at ultrasonic frequencies, is of importance to nondestructive testing, materials characterization and other research areas. Information about the decay in the coherent field due to scattering attenuation may often be used to infer information about the microstructure of the material. ${ }^{1}$ The incoherent field also contains microstructural information. Quantitative comparisons with experimental results require a well-developed model of the effects of the microstructure on the wave behavior. If the microstructure is modified, such as through the development of microcracks within the medium, this change in microstructure would manifest itself in the scattered wave fields as well. In the case of structural materials such as concrete, polycrystalline metals and most composites, these microcracks are typically induced by materials processing, manufacturing, aging and other in-service conditions. Changes in material response due to microcracking are typically inferred ultrasonically by the decrease in wave velocity or the increase in ultrasonic attenuation, both of which indicate a stiffness degradation or loss of strength of the material. ${ }^{2}$ The limits of detecting these changes in wave behavior depend on the amount of increased scattering due to the system of microcracks. Studies of elastic wave attenuation in cracked materials are found in articles by $\mathrm{Mal}^{3,4}$ Piau, ${ }^{5}$ Chatterjee et al., ${ }^{6}$ Zhang and Achenbach, ${ }^{2}$ Zhang and Gross, ${ }^{7}$ and Smyshlyaev and Willis, ${ }^{8}$ for example. This previous research has primarily been focused on specific wave/ crack interactions, rather than a more general description. More general, tensor-based, studies of elastic wave attenuation are mostly limited to polycrystalline media. ${ }^{9-13}$

In the present article, a generalized tensor-based approach is used to examine the attenuation of elastic waves in an isotropic, homogeneous medium with embedded microcracks. Here, the microcracks are assumed to be noninteract-

\footnotetext{
a) Author to whom correspondence should be addressed. Electronic mail: jaturner@unl.edu
}

ing, penny-shaped cracks that are randomly oriented. However, the coordinate-free approach allows for nonrandom ensembles of microcracks to be studied with relative ease. This topic is the subject of ongoing research by the authors. It is assumed that the constitutive behavior of the stiffness matrix or compliance matrix in the pristine state is sufficiently characterized at the local level by a linear elastic relation between the average stresses and average strains of the traditional form. In standard damage mechanics theory, ${ }^{14,15}$ the continuum model is described by a macroscopic damage parameter attributed to the microcracks.

The effective elastic moduli of the medium that contains many penny-shaped cracks is first presented using techniques discussed by Nemat-Nasser and Hori ${ }^{16}$ and Kachanov et al. ${ }^{17}$ These techniques have been used to estimate the upper and lower bounds of the material properties with distributed damage. The general inequality was presented by Hashin and Shtrikman ${ }^{18}$ using a variational approach. Similar ideas have been used to estimate the effective conductivity of such media. ${ }^{19}$ The effective Lamé constants derived here are in agreement with previous analyses. Next, expressions for the moduli fluctuations are derived in terms of the single crack compliance. The fluctuations and corresponding covariance of the moduli are necessary for the attenuation derivation. This derivation is based upon the diagrammatic approach ${ }^{12,20}$ in which the mean response is governed by the Dyson equation. The Dyson equation is then solved for the longitudinal and transverse attenuations within the limits of the first-order smoothing approximation (FOSA) ${ }^{20}$ or Keller ${ }^{21}$ approximation. The attenuations are shown to scale linearly with the damage parameter at low values. Finally, example numerical results are presented and discussed.

\section{EFFECTIVE ELASTIC PROPERTIES OF A DAMAGED ELASTIC SOLID}

Methods which make use of estimating the effective elastic properties of the statistically homogeneous elastic solids which contain a large number of microcracks are inves- 
tigated by Nemat-Nasser and Hori, ${ }^{16}$ Kachanov et al.,${ }^{17}$ and others. The first step in determining the effective properties of the damaged elastic solid involves consideration of a single penny-shaped crack, which is located within an infinite, homogeneous, isotropic and elastic continuum. In accordance with linear elastic fracture mechanics, the total, local and average stress $\overline{\boldsymbol{\sigma}}$ and strain $\bar{\gamma}$ fields hold for superposition. ${ }^{22}$ The average stress and strain are related by

$$
\bar{\gamma}=\overline{\mathbf{S}} \overline{\boldsymbol{\sigma}}, \quad \text { or } \quad \overline{\boldsymbol{\sigma}}=\overline{\mathbf{C}} \bar{\gamma},
$$

where

$$
\overline{\mathbf{S}}=\mathbf{S}+\mathbf{S}^{*}, \quad \overline{\mathbf{C}}=\mathbf{C}-\mathbf{C}^{*} .
$$

In Eqs. (1) and (2), $\bar{\gamma}=\langle\gamma(x)\rangle$ and $\overline{\boldsymbol{\sigma}}=\langle\sigma(x)\rangle$ are the ensemble average strain and average stress, respectively. Here $\overline{\mathbf{S}}$ and $\overline{\mathbf{C}}$ are the effective compliance and effective stiffness, respectively, of the elastic solid which contains the presence of the cracks. Here, $\mathbf{S}^{*}$ is defined as the effective compliance contributed by all cracks within the elastic solid and $\mathbf{C}^{*}$ is defined as the effective stiffness contributed by all cracks within the elastic solid. It should be noted that $\left(\mathbf{C}^{*}\right)^{-1}$ $\neq \mathbf{S}^{*}$. The tensors $\mathbf{S}$ and $\mathbf{C}$ are the compliance and stiffness, respectively, of the pristine material.

The components of the effective compliance tensor $\mathbf{S}^{*}$ can be estimated from the contribution by the microcracks. The contributions are calculated by the complementary strain energy in terms of the path independent integral of fracture mechanics. The strain energy is expressed as

$$
\psi^{*}=\int_{0}^{a} \frac{Q}{a} d a,
$$

where $\psi^{*}$ is the Gibbs' energy and $a$ is the crack radius. The factor $Q$ in the integrand is written as the line integral of the $J$ integral (energy release rate) along the crack perimeter $L$,

$$
Q=\oint_{L} a J d L
$$

The energy release rate $J$ is expressed in terms of the stress intensity factors $K_{m}$ ( $m=\mathrm{I}$, II,III) corresponding to the three fundamental crack modes as

$$
J=\frac{1-\nu}{2 \mu}\left(K_{\mathrm{I}}^{2}+K_{\mathrm{II}}^{2}\right)+\frac{1}{2 \mu} K_{\mathrm{III}}^{2},
$$

where $\nu$ and $\mu$ are the Poisson's ratio and shear modulus, respectively, of the surrounding material. Thus, the final Gibbs' energy is derived in compact form as

$$
\psi^{*}=\int_{0}^{a}\left[\oint \bar{M}_{m n} K_{m} K_{n} d L\right] d a .
$$

The tensor $\overline{\mathbf{M}}$ is given by

$$
\bar{M}_{m n}=\frac{1}{2 \mu}\left[(1-\nu) \delta_{m n}+\nu \delta_{m \mathrm{III}} \delta_{n \mathrm{III}}\right],
$$

where $\delta_{m n}$ is the Kronecker delta, and the subscript $n$ also represents the three fundamental cracks modes I, II, or III.

The components of the compliance tensor are related to the Gibbs' energy through $\widetilde{S}_{i j k l}^{(s)}=\partial^{2} \psi^{*} / \partial \widetilde{\sigma}_{i j} \partial \widetilde{\sigma}_{k l}$ (in the local coordinate system, the notation $\sim$ is used). Thus, the compliance attributed to the presence of a single penny-shaped active crack in a representative volume $V$ is then

$$
\widetilde{S}_{i j k l}^{(s)}=\frac{2}{V} \int_{0}^{a}\left[\oint \bar{M}_{m n} \frac{\partial K_{m}}{\partial \widetilde{\sigma}_{i j}} \frac{\partial K_{n}}{\partial \widetilde{\sigma}_{k l}} d L\right] d a .
$$

Substituting $K_{m}$ into Eq. (8) and integrating gives the compact form ${ }^{15}$

$$
\widetilde{S}_{i j k l}^{(s)}=\frac{16}{3 V} \frac{1-\widetilde{\nu}}{2-\widetilde{\nu}} \frac{1}{2 \widetilde{\mu}} a^{3}\left\{2 \widetilde{I}_{i j k l}^{5}-\widetilde{\nu} \widetilde{I}_{i j k l}^{6}\right\} .
$$

In the global coordinate system, using a coordinate transformation and assuming the normal stress at the crack surface is tensile, the effective compliance attributable to a single, planar, penny-shaped crack of radius $a$ is written in the simple form

$$
S_{i j k l}^{(s)}=\frac{16}{3 V} \frac{1-\nu}{2-\nu} \frac{1}{2 \mu} a^{3}\left\{2 I_{i j k l}^{5}-\nu I_{i j k l}^{6}\right\} .
$$

The compliance of a single crack is dependent on the unit normal $\mathbf{n}$, which defines the crack orientation. This orientation is implicit in the tensors $\mathbf{I}^{5}, \mathbf{I}^{6}$. These tensors and other necessary basis tensors are given in terms of the unit normal vector $\mathbf{n}$ and Kronecker delta $(\delta) \mathrm{as}^{23}$

$$
\begin{aligned}
& I_{i j k l}^{1}=\frac{1}{2}\left(\delta_{i k} \delta_{j l}+\delta_{i l} \delta_{j k}\right), \quad I_{i j k l}^{2}=\delta_{i j} \delta_{k l}, \\
& I_{i j k l}^{3}=\delta_{i j} n_{k} n_{l}, \quad I_{i j k l}^{4}=n_{i} n_{j} \delta_{k l}, \quad I_{i j k l}^{6}=n_{i} n_{j} n_{k} n_{l}, \\
& I_{i j k l}^{5}=\frac{1}{4}\left(n_{i} n_{k} \delta_{j l}+n_{i} n_{l} \delta_{j k}+n_{j} n_{k} \delta_{i l}+n_{j} n_{l} \delta_{i k}\right) .
\end{aligned}
$$

If it is assumed that the damaged medium is statistically homogeneous and statistically isotropic, the effective compliance attributable to a single crack is approximately the inverse of the effective stiffness. It is convenient to derive the attenuation in terms of the effective stiffness attributable to a penny-shaped crack. The compliance tensor of the pristine, undamaged elastic matrix is

$$
S_{i j k l}^{0}=\frac{1}{2 \mu}\left(I_{i j k l}^{1}-\frac{\nu}{1+\nu} I_{i j k l}^{2}\right) .
$$

Then the effective compliance expression for a simple crack in a matrix can be written

$$
\hat{\mathbf{S}}=\mathbf{S}^{(s)}+\mathbf{S}^{0}=\frac{1}{2 \mu} \sum_{i=1}^{6} e_{i} \mathbf{I}^{i},
$$

in which

$$
\begin{aligned}
& e_{1}=1, \quad e_{2}=-\frac{\nu}{1-\nu}, \quad e_{3}=e_{4}=0, \\
& e_{5}=\frac{32}{3} \frac{1-\nu}{2-\nu} a^{3}, \quad e_{6}=-\frac{16}{3} \frac{\nu(1-\nu)}{2-\nu} a^{3} .
\end{aligned}
$$

To calculate the stiffness tensor, the other irreducible tensor basis $\mathbf{J}\left(J^{i}, i=1,2, \ldots 5,6\right)$ is used. ${ }^{23}$ These basis tensors may be formed from the tensors I using a linear transformation. The effective compliance tensor expressed in terms of the $\mathbf{J}$ basis tensors is given by 


$$
\hat{\mathbf{S}}=\frac{1}{2 \mu} \sum_{i=1}^{6} f_{i} \mathbf{J}^{i}
$$

where

$$
\begin{aligned}
& f_{1}=\frac{2-\nu}{2(1+\nu)}+\frac{8}{3}(1-\nu) a^{3}, \\
& f_{2}=\frac{\nu}{2(1+\nu)}+\frac{8}{3}(1-\nu) a^{3}, \\
& f_{3}=-\frac{3 \nu}{2(1+\nu)}, \quad f_{4}=-\frac{\nu}{2(1+\nu)}, \\
& f_{5}=1+\frac{16}{3} \frac{1-\nu}{2-\nu} a^{3}, \quad f_{6}=1 .
\end{aligned}
$$

The effective stiffness tensor may also be expanded in a similar way as

$$
\hat{\mathbf{C}}=\hat{\mathbf{S}}^{-1}=2 \mu \sum_{i=1}^{6} b_{i} \mathbf{J}^{i},
$$

where the scalar coefficients $b_{i}$ are related to the scalar coefficients $f_{i}$,

$$
\left\{b_{1}, b_{2}, b_{3}, b_{4}, b_{5}, b_{6}\right\}=\Delta\left\{f_{1,}-f_{2,}-f_{3},-f_{4,} \frac{1}{f_{5} \Delta}, \frac{1}{f_{6} \Delta}\right\},
$$

with

$$
\Delta^{-1}=f_{1}^{2}-f_{2}^{2}-f_{3}^{2}+f_{4}^{2} .
$$

If the penny-shaped crack volume is much smaller than the representative volume, the higher order terms in the coefficients may be neglected. This truncation is used to simplify the form of the derivation that follows and places some limits on the resulting attenuations. In this case, the corresponding coefficients are

$$
\begin{aligned}
& b_{1}=\frac{2-\nu}{2(1-2 \nu)}+\frac{8}{3} \frac{(1-\nu)\left(-3 \nu^{2}+2 \nu-1\right)}{(1-2 \nu)^{2}} a^{3}, \\
& b_{2}=-\frac{\nu}{2(1-2 \nu)}-\frac{8}{3} \frac{(1-\nu)\left(-\nu^{2}-2 \nu+1\right)}{(1-2 \nu)^{2}} a^{3}, \\
& b_{3}=\frac{3 \nu}{2(1-2 \nu)}-\frac{8}{3} \frac{3 \nu(1-\nu)^{2}}{(1-2 \nu)^{2}} a^{3}, \\
& b_{4}=\frac{\nu}{2(1-2 \nu)}-\frac{8}{3} \frac{\nu(1-\nu)^{2}}{(1-2 \nu)^{2}} a^{3}, \\
& b_{5}=1-\frac{16}{3} \frac{1-\nu}{2-\nu} a^{3}, \quad b_{6}=1 .
\end{aligned}
$$

Again the effective stiffness is represented in terms of the six fourth order tensors $\mathbf{I}$,

$$
\hat{\mathbf{C}}=2 \mu \sum_{i=1}^{6} c_{i} \mathbf{I}^{i},
$$

where the coefficients $c_{i}$ are

$$
c_{1}=1, \quad c_{2}=\frac{\nu}{1-\nu}-\frac{16}{3} \frac{\nu^{2}(1-\nu)}{(1-2 \nu)^{2}} a^{3},
$$

$$
\begin{aligned}
& c_{3}=c_{4}=-\frac{16}{3} \frac{\nu(1-\nu)}{1-2 \nu} a^{3}, \\
& c_{5}=-\frac{32}{3} \frac{1-\nu}{2-\nu} a^{3}, \quad c_{6}=\frac{16}{3} \frac{\nu(1-\nu)}{2-\nu} a^{3} .
\end{aligned}
$$

The single crack stiffness reduces the stiffness of the pristine, undamaged elastic matrix with stiffness $\mathbf{C}^{0}=2 \mu\left(\mathbf{I}^{1}+[\nu /(1\right.$ $-\nu)] \mathbf{I}^{2}$ ). Thus, the effective stiffness of the crack in a unit volume and matrix is

$$
\mathbf{C}^{(s)}=\mathbf{C}^{0}-\hat{\mathbf{C}}=2 \mu \sum_{i=1}^{6} d_{i} \mathbf{I}^{i},
$$

where the coefficients $d_{i}$ are

$$
\begin{aligned}
& d_{1}=0, \quad d_{2}=\frac{16}{3} \frac{\nu^{2}(1-\nu)}{(1-2 \nu)^{2}} a^{3}, \\
& d_{3}=d_{4}=\frac{16}{3} \frac{\nu(1-\nu)}{1-2 \nu} a^{3}, \\
& d_{5}=\frac{32}{3} \frac{1-\nu}{2-\nu} a^{3}, \quad d_{6}=-\frac{16}{3} \frac{\nu(1-\nu)}{2-\nu} a^{3} .
\end{aligned}
$$

To estimate the ensemble average properties, an infinitely extended, homogeneous, isotropic and elastic threedimensional continuum is considered. The medium is assumed to contain a large number of microcracks which do not interact with each other. The effective compliance or stiffness may be determined by the superposition of the contributions of individual microcracks. In the case of a large number of microcracks, the summation can be replaced by an integration over a continuous distribution of crack sizes and orientations. The penny-shaped crack is characterized by its radius $a$ and two Euler angles $\theta$ and $\varphi$ that define the orientation of the unit normal $\mathbf{n}$. The specific distribution of the crack radii and orientations is expressed by the probability density function $W(a, \theta, \varphi)$. In some situations, the microcrack radii and orientations may be correlated. The density function is then replaced by the probability density function $P(a, \theta, \varphi)=P^{\theta \varphi}(\theta, \varphi) P^{a}(a \mid \theta, \varphi)$. Here, however, it is assumed that the microcrack radii and orientations are not correlated. In this case, the density function is expressed as

$$
W(a, \theta, \varphi)=A(a) \zeta(\theta, \varphi) .
$$

The effective continuum properties per unit volume are expressed in terms of an ensemble average utilizing the density function in Eqs. (25) and (23) such that

$$
C_{i j k l}^{*}=\frac{\varepsilon}{4 \pi} \int_{0}^{2 \pi} \int_{-\pi / 2}^{\pi / 2} C_{i j k l}^{(s)} \zeta(\theta, \varphi) d \theta \cos \varphi d \varphi .
$$

In Eq. (26), the nondimensional microcrack density per unit volume is defined by

$$
\varepsilon=N\left\langle a^{3}\right\rangle=\int_{a^{-}}^{a^{+}} A(a) a^{3} d a,
$$

where $N$ is number of cracks per unit volume and the angular brackets represent the ensemble average. This damage factor was introduced by Walsh ${ }^{24}$ for the case of an isotropic distribution of the penny-shaped microcracks. A more general 
form of the damage factor in terms of elliptical microcracks is given by Budiansky and O'Connell, ${ }^{25}$

$$
\varepsilon=\frac{2 N}{\pi}\left\langle\frac{\Gamma^{2}}{P}\right\rangle
$$

where $\Gamma$ and $P$ are the crack surface area and the perimeter length, respectively.

The simplest model of the microcrack distribution is assumed when their orientations are random. In this case, the normal to the microcrack plane takes every direction with equal probability, such that the effective compliance or stiffness tensor attributable to the presence of microcracks is isotropic. In this case, the density function in Eq. (25) is given by

$$
\zeta(\theta, \varphi)=1
$$

The effective stiffness attributable to the presence of $N$ active microcracks per unit volume is then derived from Eq. (26),

$$
C_{i j k l}^{*}=\frac{\varepsilon}{4 \pi} \int_{0}^{2 \pi} \int_{-\pi / 2}^{\pi / 2} C_{i j k l}^{(s)}(\theta, \varphi) \cos \varphi d \theta d \varphi,
$$

where the $C_{i j k l}^{(s)}$ is expressed in Eq. (23). By integrating the expression in Eq. (30), the effective stiffness due to an isotropic distribution of penny shaped microcracks is derived as

$$
\begin{aligned}
C_{i j k l}^{*}= & \frac{16}{45} \frac{1-\nu}{2-\nu} 2 \mu \varepsilon\left\{2(5-\nu) I_{i j k l}^{1}\right. \\
& \left.+\frac{\nu\left(\nu^{2}-16 \nu+19\right)}{(1-2 \nu)^{2}} I_{i j k l}^{2}\right\} .
\end{aligned}
$$

In addition, the stiffness tensor of the homogeneous, isotropic and elastic solids in its pristine, undamaged state is

$$
C_{i j k l}^{0}=\lambda I_{i j k l}^{2}+2 \mu I_{i j k l}^{1} \text {. }
$$

Here, the ensemble average stiffness is redefined such that the average fluctuations are zero. Such a procedure, while not necessary, is convenient for the calculation of material covariance and attenuation. The moduli are assumed to be spatially varying and of the form

$$
\bar{C}_{i j k l}(\mathbf{x})=\bar{C}_{i j k l}^{0}+\delta \bar{C}_{i j k l}(\mathbf{x}),
$$

where

$$
\bar{C}_{i j k l}^{0}=C_{i j k l}^{0}-C_{i j k l}^{*} .
$$

Thus, the moduli have the form of the average moduli

$$
\bar{C}_{i j k l}^{0}=\left\langle\bar{C}_{i j k l}(\mathbf{x})\right\rangle=\bar{\lambda} I_{i j k l}^{2}+2 \bar{\mu} I_{i j k l}^{1},
$$

plus the fluctuation about the mean $\delta \bar{C}_{i j k l}$. Hence, $\bar{C}_{i j k l}$ has the average value of $\bar{C}_{i j k l}^{0}$ and $\delta \bar{C}_{i j k l}$ represents the modulus fluctuation. The effective constants are

$$
\begin{aligned}
& \bar{\mu}=\mu\left[1-\frac{32}{45} \frac{(1-\nu)(5-\nu)}{2-\nu} \varepsilon\right], \\
& \bar{\lambda}=\lambda\left[1-\frac{16}{45} \frac{(1-\nu)\left(\nu^{2}-16 \nu+19\right)}{(2-\nu)(1-2 \nu)} \varepsilon\right] .
\end{aligned}
$$

These results are identical with the results obtained by Krajcinovic, ${ }^{15}$ Kachanov et al., ${ }^{26}$ Budiansky and O'Connell, ${ }^{25}$ and Zimmerman. ${ }^{27}$ The effective properties of the damaged material are shown by Eqs. (36) to be linearly related to the damage parameter $\varepsilon$. Thus, wave speed changes that are the result of damage will scale linearly with $\varepsilon$ as well.

The fluctuations, which are defined here to have zero average, $\langle\delta \bar{C}\rangle=0$, are given by

$$
\delta \bar{C}_{i j k l}(\mathbf{x})=C_{i j k l}^{*}-C_{i j k l}^{(s)} H(\mathbf{x}) .
$$

The function $H(\mathbf{x})$ is defined as

$$
H(\mathbf{x})= \begin{cases}1 & \text { if } \mathbf{x} \in \mathbf{S}, \\ 0 & \text { otherwise, }\end{cases}
$$

where $\mathbf{S}$ is the spatial volume occupied by a crack.

\section{ENSEMBLE AVERAGE RESPONSE}

The propagation and scattering of elastic waves in heterogeneous media is presented in this section in terms of the Dyson equation. Such an approach has been discussed by Frisch $^{20}$ and used by others. ${ }^{12,13}$ This section is primarily included for completeness. The equation of motion for the elastodynamic response of a linear, elastic solid to deformation is given in terms of the Green's dyadic by

$$
\begin{gathered}
\left\{-\delta_{j k} \rho(\mathbf{x}) \partial_{t}^{2}+\partial x_{i} \bar{C}_{i j k l}(\mathbf{x}) \partial x_{l}\right\} G_{k \alpha}\left(\mathbf{x}, \mathbf{x}^{\prime} ; t\right) \\
=\delta_{j \alpha} \delta^{3}\left(\mathbf{x}-\mathbf{x}^{\prime}\right) \delta(t),
\end{gathered}
$$

where $\delta^{3}\left(\mathbf{x}-\mathbf{x}^{\prime}\right)$ is the three-dimensional spatial Dirac delta function. The second order Green's dyadic, $G_{k \alpha}\left(\mathbf{x}, \mathbf{x}^{\prime} ; t\right)$, is defined as the displacement response at location $\mathbf{x}$ in the $k$ th direction due to an unit impulsive force acting at position $\mathbf{x}^{\prime}$ in the $\alpha$ th direction at time zero. The moduli are considered to vary spatially whereas the density is assumed uniform throughout with units chosen such that the density is unity $[\rho(\mathbf{x})=1]$. The effective fourth-rank stiffness tensor $\bar{C}_{i j k l}(\mathbf{x})$ is given in Eq. (33). It is assumed that the fluctuations are small, such that $\delta \overline{\mathbf{C}} \ll \overline{\mathbf{C}}^{0}$. The covariance of the moduli is characterized by an eighth rank tensor

$$
\left\langle\delta C_{i j k l}(\mathbf{x}) \delta C_{\alpha \beta \gamma \delta}(\mathbf{y})\right\rangle=\Xi_{i j k l}^{\alpha \beta \gamma \delta} \eta(|\mathbf{x}-\mathbf{y}|) .
$$

The spatial and tensorial parts of the covariance, $\eta$ and $\Xi$, respectively, are assumed independent. Also, $\eta$ is assumed to be a function of the magnitude of the difference between two vectors, $|\mathbf{x}-\mathbf{y}|$, rather than $\mathbf{x}$ and $\mathbf{y}$ separately. This assumption implies that the medium is statistically homogeneous and statistically isotropic.

The spatio-temporal Fourier transform pair for the function $f(\mathbf{x}, t)$ and $\widetilde{f}(\mathbf{p}, \omega)$ is defined as

$$
\begin{aligned}
& \widetilde{f}(\mathbf{p}, \omega)=\int_{-\infty}^{+\infty} \int_{-\infty}^{+\infty} f(\mathbf{x}, t) e^{i \omega t} e^{-i \mathbf{x} \cdot \mathbf{p}} d^{3} x d t, \\
& f(\mathbf{x}, t)=\frac{1}{(2 \pi)^{4}} \int_{-\infty}^{+\infty} \int_{-\infty}^{+\infty} \widetilde{f}(\mathbf{p}, \omega) e^{-i \omega t} e^{i \mathbf{x} \cdot \mathbf{p}} d^{3} p d \omega .
\end{aligned}
$$

These definitions allow Eq. (39) to be temporally transformed in the following form: 


$$
\begin{aligned}
& \left\{\omega^{2} \delta_{j k}+\bar{C}_{i j k l}^{0} \partial_{i} \partial_{l}+\partial_{i} \delta \bar{C}_{i j k l}(\mathbf{x}) \partial_{l}\right\} \mathbf{G}_{k \alpha}\left(\mathbf{x}, \mathbf{x}^{\prime} ; \omega\right) \\
& =\delta_{j \alpha} \delta^{3}\left(\mathbf{x}-\mathbf{x}^{\prime}\right) .
\end{aligned}
$$

The mean response is governed by the Dyson equation ${ }^{12,20}$

$$
\begin{aligned}
\left\langle G_{i \alpha}\left(\mathbf{x}, \mathbf{x}^{\prime}\right)\right\rangle= & G_{i \alpha}^{0}\left(\mathbf{x}, \mathbf{x}^{\prime}\right)+\iint G_{i \beta}^{0}(\mathbf{x}, \mathbf{y}) M_{\beta j}(\mathbf{y}, \mathbf{z}) \\
& \times\left\langle G_{j \alpha}\left(\mathbf{z}, \mathbf{x}^{\prime}\right)\right\rangle d^{3} y d^{3} z .
\end{aligned}
$$

The notation $G_{i \alpha}^{0}\left(\mathbf{x}, \mathbf{x}^{\prime}\right)$ is the bare Green's dyadic defined as the ensemble average response of the medium (without fluctuations), namely, the solution to Eq. (42) when $\delta \bar{C}_{i j k l}(\mathbf{x})$ $=0$. The second order tensor $\mathbf{M}$ is the mass (self-energy) operator. Equation (43) is easily solved in the Fourier transform domain under the assumption of statistical homogeneity. The spatial Fourier transform pair for $\mathbf{G}^{0}$ is first defined as

$$
\begin{aligned}
G_{i \alpha}^{0}(\mathbf{p}) \delta^{3}(\mathbf{p}-\mathbf{q})= & \frac{1}{(2 \pi)^{3}} \iint G_{i \alpha}^{0}\left(\mathbf{x}, \mathbf{x}^{\prime}\right) \\
& \times e^{-i \mathbf{p} \cdot \mathbf{x}} e^{i \mathbf{q} \cdot \mathbf{x}^{\prime}} d^{3} x d^{3} x^{\prime}, \\
G_{i \alpha}^{0}\left(\mathbf{x}, \mathbf{x}^{\prime}\right)= & \frac{1}{(2 \pi)^{3}} \iint G_{i \alpha}^{0}(\mathbf{p}) \delta^{3}(\mathbf{p}-\mathbf{q}) \\
& \times e^{i \mathbf{p} \cdot \mathbf{x}} e^{-i \mathbf{q} \cdot \mathbf{x}^{\prime}} d^{3} p d^{3} q .
\end{aligned}
$$

The bare Green's dyadic, $\mathbf{G}^{0}$, is the solution of Eq. (42) with the modulus fluctuation equal to zero. Hence,

$$
\mathbf{G}^{0}(\mathbf{p})=\hat{\mathbf{p}} \hat{\mathbf{p}} g_{L}^{0}(p)+(\mathbf{I}-\hat{\mathbf{p}} \hat{\mathbf{p}}) g_{T}^{0}(p),
$$

for propagation in the $\hat{\mathbf{p}}$ direction. The bare longitudinal wave $g_{L}^{0}(p)$ and transverse wave $g_{T}^{0}(p)$ propagators are denoted

$$
\begin{aligned}
& g_{L}^{0}(p)=\left[\omega^{2}-p^{2} c_{L}^{2}\right]^{-1}, \\
& g_{T}^{0}(p)=\left[\omega^{2}-p^{2} c_{T}^{2}\right]^{-1},
\end{aligned}
$$

where the $c_{L}$ and $c_{T}$ are the average longitudinal and transverse wave speeds, respectively. The Fourier transforms which define $\langle\mathbf{G}(\mathbf{p})\rangle$ and $\tilde{\mathbf{M}}(\mathbf{p})$ are given by expressions similar to those defining $\mathbf{G}^{0}(\mathbf{p})$. The assumption of statistical homogeneity ensures that they are functions of a single wave vector in Fourier space. The Dyson equation is then transformed and solved to give

$$
\langle\mathbf{G}(\mathbf{p})\rangle=\left[\mathbf{G}^{0}(\mathbf{p})^{-1}-\tilde{\mathbf{M}}(\mathbf{p})\right]^{-1} .
$$

Here $\widetilde{\mathbf{M}}(\mathbf{p})$ is the spatial transform of the self-energy. The self-energy, in turn, can be written as an expansion in powers of material property fluctuations. An approximation of $\mathbf{M}$ is made to first order using the first term in such an expansion. $^{20,21}$ To this level of approximation, $\mathbf{M}$ is expressed as $^{12}$

$$
\begin{aligned}
& M_{\beta j}(\mathbf{y}, \mathbf{z}) \\
& \quad \approx\left\langle\frac{\partial}{\partial y_{\alpha}} \delta \bar{C}_{\alpha \beta \gamma \delta}(\mathbf{y}) \frac{\partial}{\partial y_{\delta}} \mathbf{G}_{\gamma k}^{0}(\mathbf{y}, \mathbf{z}) \frac{\partial}{\partial z_{i}} \delta \bar{C}_{i j k l}(\mathbf{z}) \frac{\partial}{\partial z_{l}}\right\rangle .
\end{aligned}
$$

The approximation is valid if the fluctuations are not too large. The spatial Fourier transform defined in Eq. (44) allows the transform of the self-energy to be written as a convolution

$$
\widetilde{M}_{\beta j}(\mathbf{p})=\int d^{3}{ }_{s} G_{\gamma k}^{0}(\mathbf{s}) p_{\alpha} p_{l} s_{\delta} s_{i} \Xi_{i j k l}^{\alpha \beta \gamma \delta} \widetilde{\eta}(\mathbf{p}-\mathbf{s}) .
$$

The spatial transform of the self-energy and the mean Green's dyadic have the same form as the bare Green's dyadic. Hence, one may write

$$
\begin{aligned}
& \tilde{\mathbf{M}}(\mathbf{p})=\hat{\mathbf{p}} \hat{\mathbf{p}} m_{L}(p)+(\mathbf{I}-\hat{\mathbf{p}} \hat{\mathbf{p}}) m_{T}(p), \\
& \langle\widetilde{\mathbf{G}}(\mathbf{p})\rangle=\hat{\mathbf{p}} \hat{\mathbf{p}} g_{L}(p)+(\mathbf{I}-\hat{\mathbf{p}} \hat{\mathbf{p}}) g_{T}(p),
\end{aligned}
$$

where

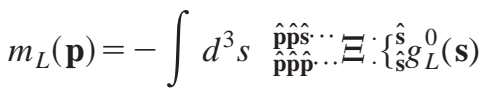

$$
\begin{aligned}
& \left.+\left(\mathbf{I}-{ }_{\hat{\mathbf{s}}}^{\hat{\mathbf{s}}}\right) g_{T}^{0}(\mathbf{s})\right\} \widetilde{\eta}(\mathbf{p}-\mathbf{s}),
\end{aligned}
$$

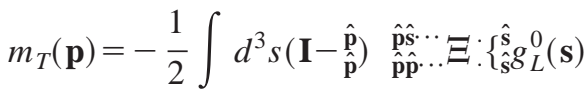

$$
\begin{aligned}
& \left.+\left(\mathbf{I}-{ }_{\hat{\mathbf{s}}}^{\hat{\mathbf{s}}}\right) g_{T}^{0}(\mathbf{s})\right\} \widetilde{\eta}(\mathbf{p}-\mathbf{s})
\end{aligned}
$$

and

$$
\begin{aligned}
& g_{L}(p)=\left[\omega^{2}-p^{2} c_{L}^{2}+m_{L}(p)\right]^{-1}, \\
& g_{T}(p)=\left[\omega^{2}-p^{2} c_{T}^{2}+m_{T}(p)\right]^{-1} .
\end{aligned}
$$

These are the expressions for the mean response. They define the phase velocity and the attenuation of longitudinal and transverse wave types. The inverse Fourier transform of $\langle\mathbf{G}(\mathbf{p})\rangle$ in Eq. (50) is dominated by the poles near $|p|$ $=\omega / c_{\beta}$ with $\beta=L$ or $T$. Thus, the effective wave vectors, $\mathbf{p}$, are the solution to the required conditions

$$
\omega^{2}-p^{2} c_{L}^{2}+m_{L}(p)=0
$$

and

$$
\omega^{2}-p^{2} c_{T}^{2}+m_{T}(p)=0 .
$$

The real and imaginary parts of $\mathbf{p}$ give the phase velocity and attenuation, respectively. Equations (53) and (54) are usually solved numerically using root finding techniques. The solution for the attenuation can be calculated by making use of an approximation valid below the high frequency geometric optics limit. In this case, the solutions, $\mathbf{p}$, are approximated as equal to the bare solution. Such an approximation, $m(\mathbf{p})$ $\approx m\left(\omega \hat{\mathbf{p}} / c_{\beta}\right)$ is sometimes called a Born approximation. In essence, the phase velocity is supposed to remain unchanged by the heterogeneities. Under this approximation the imaginary part can be evaluated from Eqs. (53) and (54). The attenuations, which are the imaginary part for each wave type, are calculated as

$$
\alpha_{\beta}(\hat{\mathbf{p}})=-\frac{1}{2 \omega c_{\beta}} \operatorname{Im} m_{\beta}\left(\frac{\omega}{c_{\beta}} \hat{\mathbf{p}}\right) .
$$

The imaginary parts of $m_{\beta}$ (where $\beta=L$ or $T$ ) are determined from Eqs. (51) such that the attenuations are given by 


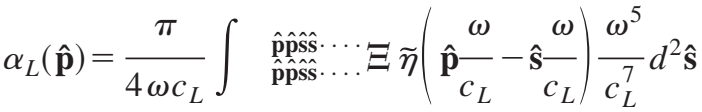

$$
\begin{aligned}
& +\frac{\pi}{4 \omega c_{T}} \int \underset{\hat{\mathbf{p}} \hat{\mathbf{p}} \hat{\mathbf{s}}}{\hat{\mathbf{p}} \hat{\mathbf{s}}}\left(\mathbf{I}-{ }_{\hat{\mathbf{s}}}^{\hat{\mathbf{s}}}\right) \ldots \ldots \Xi \widetilde{\eta}\left(\hat{\mathbf{p}} \frac{\omega}{c_{L}}\right. \\
& \left.-\hat{\mathbf{s}} \frac{\omega}{c_{T}}\right) \frac{\omega^{5}}{c_{T}^{5} c_{L}^{2}} d^{2} \hat{\mathbf{s}}
\end{aligned}
$$

and

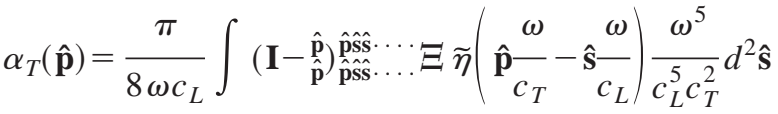

$$
\begin{aligned}
& +\frac{\pi}{8 \omega c_{T}} \int(\mathbf{I}-\underset{\hat{\mathbf{p}}}{\hat{\mathbf{p}}}) \hat{\mathbf{p} \hat{\mathbf{p}}} \hat{\mathbf{s}}(\mathbf{I}-\underset{\hat{\mathbf{s}}}{\hat{\mathbf{s}}}) \ldots \ldots \\
& \times \widetilde{\eta}\left(\hat{\mathbf{p}} \frac{\omega}{c_{T}}-\hat{\mathbf{s}} \frac{\omega}{c_{T}}\right) \frac{\omega^{5}}{c_{T}^{7}} d^{2} \hat{\mathbf{s}},
\end{aligned}
$$

where the integrals are over the unit sphere $\hat{\mathbf{s}}$. Three additional functions are defined as

$$
\begin{aligned}
& \eta_{L L}\left(\theta_{p s}\right)=\widetilde{\eta}\left(\hat{\mathbf{p}} \frac{\omega}{c_{L}}-\hat{\mathbf{s}} \frac{\omega}{c_{L}}\right), \\
& \eta_{T T}\left(\theta_{p s}\right)=\widetilde{\eta}\left(\hat{\mathbf{p}} \frac{\omega}{c_{T}}-\hat{\mathbf{s}} \frac{\omega}{c_{T}}\right), \\
& \eta_{L T}\left(\theta_{p s}\right)=\eta_{T L}\left(\theta_{p s}\right)=\widetilde{\eta}\left(\hat{\mathbf{p}} \frac{\omega}{c_{L}}-\hat{\mathbf{s}} \frac{\omega}{c_{T}}\right),
\end{aligned}
$$

where the direction $\hat{\mathbf{p}}$ defines the propagation direction, $\hat{\mathbf{s}}$ defines the scattered direction, and $\theta_{p s}$ is the angle between these directions (i.e., $\cos \theta_{p s}=\hat{\mathbf{p}} \cdot \hat{\mathbf{s}}$ ).

\section{ATTENUATIONS AND COVARIANCE}

To derive the attenuations, the relevant inner products on the covariance of the effective moduli fluctuations are required. The covariance of the moduli fluctuations is represented by an eighth-rank tensor which is given in Eq. (40). The inner product is given explicitly by

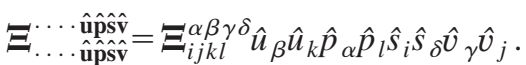

The covariance here is given in terms of an average over all crack orientations. Thus, the crack normal $\mathbf{n}$ may vary over all possible directions. In this case, the following identities are needed:

$$
\begin{aligned}
& \left\langle n_{i} n_{j}\right\rangle=\frac{1}{3} \delta_{i j}, \\
& \left\langle n_{i} n_{j} n_{k} n_{l}\right\rangle=\frac{1}{15}\left(\delta_{i j} \delta_{k l}+\delta_{i k} \delta_{j l}+\delta_{i l} \delta_{j k}\right), \\
& \left\langle n_{i} n_{j} n_{k} n_{l} n_{\alpha} n_{\beta}\right\rangle=\frac{1}{105}\left(\delta_{i j} \delta_{k l} \delta_{\alpha \beta}+\right.\text { all permutations } \\
& -15 \text { terms in all), } \\
& \left\langle n_{i} n_{j} n_{k} n_{l} n_{\alpha} n_{\beta} n_{\gamma} n_{\delta}\right\rangle=\frac{1}{945}\left(\delta_{i j} \delta_{k l} \delta_{\alpha \beta} \delta_{\gamma \delta}\right. \\
& + \text { all permutations } \\
& -105 \text { terms in all), }
\end{aligned}
$$

where the brackets, \langle\rangle , denote the ensemble average. All averages of odd numbers of n's are zero. The average of the tensorial part of the covariance over all orientations of crack normal is defined by

$$
\Xi_{i j k l}^{\alpha \beta \gamma \delta}=\frac{1}{4 \pi} \int_{0}^{2 \pi} \int_{-\pi / 2}^{\pi / 2} \bar{C}_{i j k l}^{(s)} \bar{C}_{\alpha \beta \gamma \delta}^{(s)} \cos \varphi d \varphi d \theta .
$$

Here, we use the relation $\langle H(\mathbf{x}) H(\mathbf{y})\rangle=\varepsilon \operatorname{Pr}(r \mid 0)$, where $\operatorname{Pr}(r \mid 0)=(1-\varepsilon) \eta(r)+\varepsilon$ is defined as the conditional probability. ${ }^{28}$ The notation $r$ is used to denote the magnitude of the difference between two vectors, $\mathbf{x}$ and $\mathbf{y}$. Here, the second order terms are neglected under the assumption that the damage density is small. Thus, $\left\langle\delta C_{i j k l}(\mathbf{x}) \delta C_{\alpha \beta \gamma \delta}(\mathbf{y})\right\rangle$ $=\varepsilon \eta(r) \Xi_{i j k l}^{\alpha \beta \gamma \delta}$. It is noted that

$$
\overline{\mathbf{C}}^{(s)}=2 \mu \sum_{i=1}^{6} \bar{d}_{i} \mathbf{I}^{i},
$$

with

$$
\begin{aligned}
& \bar{d}_{1}=0, \quad \bar{d}_{2}=\frac{16}{3} \frac{\nu^{2}(1-\nu)}{(1-2 \nu)^{2}}, \quad \bar{d}_{5}=\frac{32}{3} \frac{1-\nu}{2-\nu}, \\
& \bar{d}_{3}=\bar{d}_{4}=\frac{16}{3} \frac{\nu(1-\nu)}{1-2 \nu}, \quad \bar{d}_{6}=-\frac{16}{3} \frac{\nu(1-\nu)}{2-\nu} .
\end{aligned}
$$

Using the identities in Eqs. (58), the general form of $\Xi$ is given in terms of Kronecker deltas. The expressions for the attenuations, in turn, involve certain inner products of $\Xi$ with incoming and outgoing wave vectors. In terms of the angle between $\hat{\mathbf{p}}$ and $\hat{\mathbf{s}}$, these necessary inner products reduce to

$$
\begin{aligned}
& \Xi^{\cdots} \cdots \hat{\hat{p} \hat{p} \hat{p} \hat{\mathbf{p}} \hat{\mathbf{s}} \mathbf{s}}=\left[T_{1}+4 T_{4}+4 T_{7}\right]+\left[4 T_{2}+4 T_{4}\right. \\
& \left.+32 T_{5}+16 T_{6}+16 T_{7}\right] \cos ^{2} \theta_{p s} \\
& +\left[4 T_{3}+16 T_{6}+4 T_{7}\right] \cos ^{4} \theta_{p s}, \\
& \Xi_{\cdots \cdots \hat{\mathbf{p}} \hat{\mathbf{p}} \hat{\mathbf{s}} \hat{\mathbf{s}}_{2}} \hat{\mathbf{p}}_{\mathbf{s}}=\Xi_{2} \cdots \hat{\mathbf{p}}_{2} \hat{\mathbf{p} \hat{\mathbf{s}} \hat{\mathbf{s}}} \\
& =\left[T_{4}+2 T_{7}\right]+\left[4 T_{5}+4 T_{6}+4 T_{7}\right] \cos ^{2} \theta_{p s},
\end{aligned}
$$

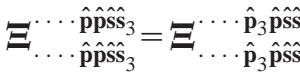

$$
\begin{aligned}
& =\left[T_{4}+4 T_{5}+4 T_{6}+6 T_{7}\right] \\
& +\left[4 T_{3}+16 T_{6}+4 T_{7}\right] \cos ^{2} \theta_{p s} \\
& -\left[4 T_{3}+16 T_{6}+4 T_{7}\right] \cos ^{4} \theta_{p s} \text {, }
\end{aligned}
$$

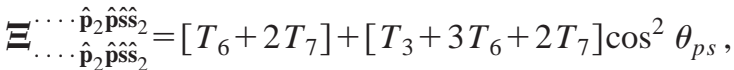

$$
\begin{aligned}
& \Xi_{\cdots \cdots \hat{\mathbf{p}}_{3} \hat{\mathbf{p}} \hat{\mathbf{p}} \hat{\mathbf{s}} \hat{\mathbf{s}}_{3}}=\left[T_{3}+4 T_{6}+4 T_{7}\right] \\
& +\left[-4 T_{3}-16 T_{6}-4 T_{7}\right] \cos ^{2} \theta_{p s} \\
& +\left[4 T_{3}+16 T_{6}+4 T_{7}\right] \cos ^{4} \theta_{p s},
\end{aligned}
$$

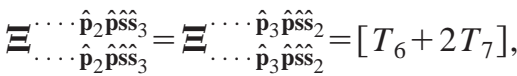




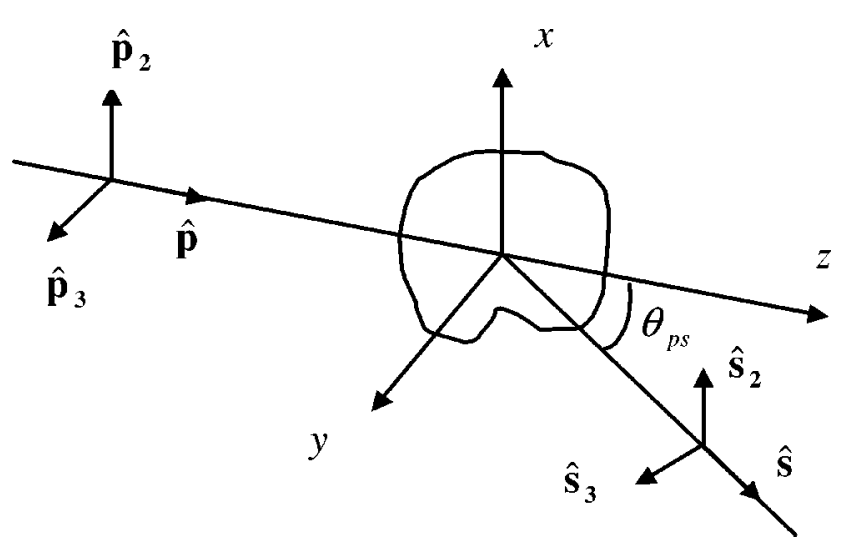

FIG. 1. Geometry for scattering in the local coordinate system.

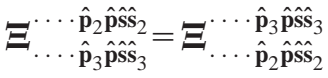

$$
\begin{aligned}
& =\left[-T_{3}-2 T_{6}\right] \cos \theta_{p s} \\
& +\left[2 T_{3}+4 T_{6}\right] \cos ^{3} \theta_{p s}, \\
& \Xi_{\cdots \cdots \hat{\mathbf{p}}_{2} \hat{\mathbf{p}} \hat{\mathbf{s}} \hat{\mathbf{s}}_{3}} \boldsymbol{\Xi}_{3} \hat{\mathbf{p}}^{\mathbf{s} \mathbf{s}_{2}}=\Xi^{\cdots} \cdots \hat{\mathbf{p}}_{3} \hat{\mathbf{p}} \hat{\mathbf{s}} \hat{\mathbf{s}}_{2} \\
& =-3 T_{6} \cos \theta_{p s}+\left[4 T_{6}+2 T_{7}\right] \cos ^{3} \theta_{p s},
\end{aligned}
$$

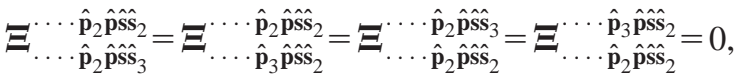

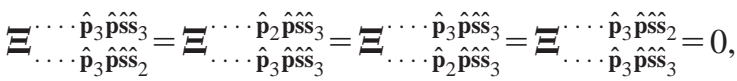

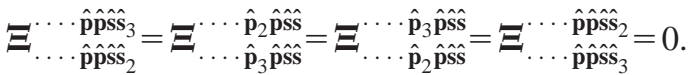

The coefficients $T_{i}$ are given by

$$
\begin{aligned}
T_{1} & =-\frac{D^{2} \nu^{2}}{945} \frac{335 \nu^{4}-1984 \nu^{3}+2946 \nu^{2}-208 \nu-937}{(1-2 \nu)^{2}} \\
T_{2}= & \frac{D^{2} \nu}{4725} \frac{31 \nu^{3}-139 \nu^{2}+\nu+243}{1-2 \nu} \\
T_{7}= & \frac{D^{2} \nu^{2}(1-2 \nu)^{2}}{945} \\
T_{3}= & \frac{D^{2}}{945}(1-2 \nu)^{2}\left(\nu^{2}-18 \nu+63\right), \\
T_{4}= & \frac{D^{2} \nu^{2}}{945}\left(31 \nu^{2}-166 \nu+217\right), \\
T_{5}= & \frac{D^{2} \nu}{1890}(1-2 \nu)\left(14 \nu^{2}-79 \nu+117\right), \\
T_{6}= & \frac{D^{2}}{420}(1-2 \nu)^{2}(7-4 \nu),
\end{aligned}
$$

with the constant $D=\frac{16}{3}[(1-\nu) /(2-\nu)(1-2 \nu)] 2 \mu$. The unit vectors $\hat{\mathbf{p}}, \hat{\mathbf{p}}_{2}, \hat{\mathbf{p}}_{3}$ and $\hat{\mathbf{s}}, \hat{\mathbf{s}}_{2}, \hat{\mathbf{s}}_{3}$ used in Eqs. (62) are defined as orthonormal triads, respectively (see Fig. 1). The general compact form of $\Xi$ is not given here. The interested reader may contact the authors for further details.

Using the notation

$$
\begin{aligned}
& F\left(\theta_{p s}\right)=\Xi \begin{array}{l}
\cdots \hat{\mathbf{p}} \hat{\mathbf{p}} \hat{\mathbf{s}} \mathbf{s} \\
\cdots \cdot \hat{\mathbf{p}} \hat{\mathbf{p}} \hat{\mathbf{s}}
\end{array}
\end{aligned}
$$

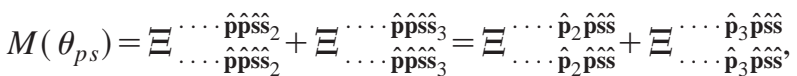

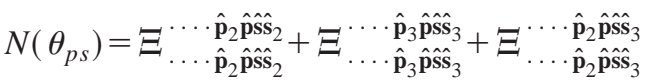

$$
\begin{aligned}
& +\Xi \cdots \hat{\mathbf{p}}_{3} \hat{\mathbf{p}} \hat{\mathbf{s}}_{2}
\end{aligned}
$$

allows the attenuations to be reduced to the form

$$
\begin{aligned}
\alpha_{L L} & =\frac{1}{4} \frac{\pi \omega^{4} \varepsilon}{c_{L}^{8}} \int \eta^{L L}\left(\theta_{p s}\right) F\left(\theta_{p s}\right) d^{2} \hat{\mathbf{s}} \\
& =\frac{1}{2} \frac{\pi^{2} \omega^{4} \varepsilon}{c_{L}^{8}} \int_{-1}^{+1} \eta^{L L}(\theta) F(\theta) d \cos \theta, \\
\alpha_{L T} & =\frac{1}{4} \frac{\pi \omega^{4} \varepsilon}{c_{L}^{3} c_{T}^{5}} \int \eta^{L T}\left(\theta_{p s}\right) M\left(\theta_{p s}\right) d^{2} \hat{\mathbf{s}} \\
& =\frac{1}{2} \frac{\pi^{2} \omega^{4} \varepsilon}{c_{L}^{3} c_{T}^{5}} \int_{-1}^{+1} \eta^{L T}(\theta) M(\theta) d \cos \theta, \\
\alpha_{T L} & =\frac{1}{2}\left(\frac{c_{T}}{c_{L}}\right)^{2} \alpha_{L T}, \\
\alpha_{T T} & =\frac{1}{8} \frac{\pi \omega^{4} \varepsilon}{c_{T}^{8}} \int^{T T}\left(\theta_{p s}\right) N\left(\theta_{p s}\right) d^{2} \hat{\mathbf{s}} \\
& =\frac{1}{4} \frac{\pi^{2} \omega^{4} \varepsilon}{c_{T}^{8}} \int_{-1}^{+1} \eta^{T T}(\theta) N(\theta) d \cos \theta .
\end{aligned}
$$

The total attenuations for the longitudinal and transverse waves are given by

$$
\alpha_{L}=\alpha_{L L}+\alpha_{L T}, \quad \alpha_{T}=\alpha_{T T}+\alpha_{T L} .
$$

The appropriate expressions for the tensorial part of the covariance, Eqs. (62), and the final expressions for attenuations, Eqs. (67) and (68), are the main results of this section. The attenuations are expressed as integrations on the unit circle in terms of the spatial transform of the spatial correlation function. Most importantly, the results are expressed in terms of the damage density $\varepsilon$. We see that the attenuations scale linearly with damage factor $\varepsilon$. In the next section, a form for $\eta$ is assumed and example results presented.

\section{EXAMPLE RESULTS}

In this section, example results are presented in terms of the dimensionless damage parameter $\varepsilon$ for an assumed spatial correlation function. For the example case, the material properties of the uncracked medium used are Young's modulus $E=2.0 \times 10^{7} \mathrm{~Pa}$ and Poisson's ratio $\nu=0.16$.

As discussed following Eq. (40), the tensorial and spatial contributions of the material covariance are assumed independent. The spatial correlations were characterized by $\eta$. Here it is assumed that $\eta$ has an exponential form

$$
\eta(r)=e^{-r / L},
$$

where $L$ is the spatial correlation length, $L=2\langle a\rangle$. As discussed by Stanke, ${ }^{29}$ an exponential function describes the 
correlation of continuous and discrete materials reasonably well. Such a model, with a single length scale, is perhaps oversimplified for materials containing a wide range of crack sizes. However, for many materials, such a model is expected to described the statistics of the material properties well. Other correlation functions, such as that discussed by Markov and Willis, ${ }^{28}$ are thought to give similar results for the frequency range considered here. The influence of this choice of correlation function on the attenuations is the subject of future investigations.

In transform space

$$
\widetilde{\eta}(p)=\frac{L^{3}}{\pi^{2}\left(1+L^{2} p^{2}\right)^{2}} .
$$

With the length scale of the spatial correlation introduced, dimensionless longitudinal and transverse frequencies are defined as $x_{L}=\omega L / c_{L}$ and $x_{T}=\omega L / c_{T}$. The transform of the difference between two wave vectors is then expressed as

$$
\eta_{\alpha \beta}(\chi)=\frac{L^{3}}{\pi^{2}\left(1+x_{\alpha}^{2}+x_{\beta}^{2}-2 x_{\alpha} x_{\beta} \chi\right)^{2}} .
$$

Here the scripts, $\alpha, \beta$ denote the wave types $L$ or $T$, and $\chi=\cos \theta_{p s}$. In dimensionless form, the attenuations in Eqs. (67) simplify to

$$
\begin{aligned}
& \alpha_{L L} H=\frac{x_{L}^{4} B^{4} \varepsilon}{2 \mu^{2}} \int_{-1}^{+1} \frac{h_{1}+h_{2} \chi^{2}+h_{3} \chi^{4}}{\left(1+2 x_{L}^{2}(1-\chi)\right)^{2}} d \chi, \\
& \alpha_{L T} H=\frac{x_{L}^{4} \varepsilon}{2 B \mu^{2}} \int_{-1}^{+1} \frac{m_{1}+m_{2} \chi^{2}+m_{3} \chi^{4}}{\left(1+x_{L}^{2}+x_{T}^{2}-2 x_{L} x_{T} \chi\right)^{2}} d \chi, \\
& \alpha_{T T} H=\frac{x_{T}^{4} \varepsilon}{4 \mu^{2}} \int_{-1}^{+1} \frac{n_{1}+n_{2} \chi^{2}+n_{3} \chi^{4}}{\left(1+2 x_{T}^{2}(1-\chi)\right)^{2}} d \chi,
\end{aligned}
$$

where $B=c_{T} / c_{L}$ is the wave speed ratio. The coefficients $h_{i}, m_{i}, n_{i}(i=1,2,3)$ are given as

$$
\begin{aligned}
& h_{1}=T_{1}+4 T_{4}+4 T_{7}, \\
& h_{2}=4 T_{2}+4 T_{4}+32 T_{5}+16 T_{6}+16 T_{7}, \\
& h_{3}=4 T_{3}+16 T_{6}+4 T_{7}, \\
& m_{1}=2 T_{4}+4 T_{5}+4 T_{6}+8 T_{7}, \\
& m_{2}=4 T_{3}+4 T_{5}+20 T_{6}+8 T_{7}, \\
& m_{3}=-4 T_{3}-16 T_{6}-4 T_{7}, \\
& n_{1}=T_{3}+7 T_{6}+10 T_{7}, \\
& n_{2}=-3 T_{3}-13 T_{6}-2 T_{7}, \\
& n_{3}=4 T_{3}+16 T_{6}+4 T_{7} .
\end{aligned}
$$

Example calculations using Eqs. (72)-(74) are shown in Fig. 2. The dimensionless longitudinal and transverse attenuations, $\alpha_{L} H$ and $\alpha_{T} H$, respectively, are plotted as a function of dimensionless frequency, $x_{L}$, for two values of the damage parameter $\varepsilon$. Equations (72)-(74) are shown to scale linearly with $\varepsilon$ such that other values of $\varepsilon$ lead to constant shifts in these curves. The attenuations increase with the fourth power of frequency in the low frequency limit as expected. After a transition region, the attenuations increase

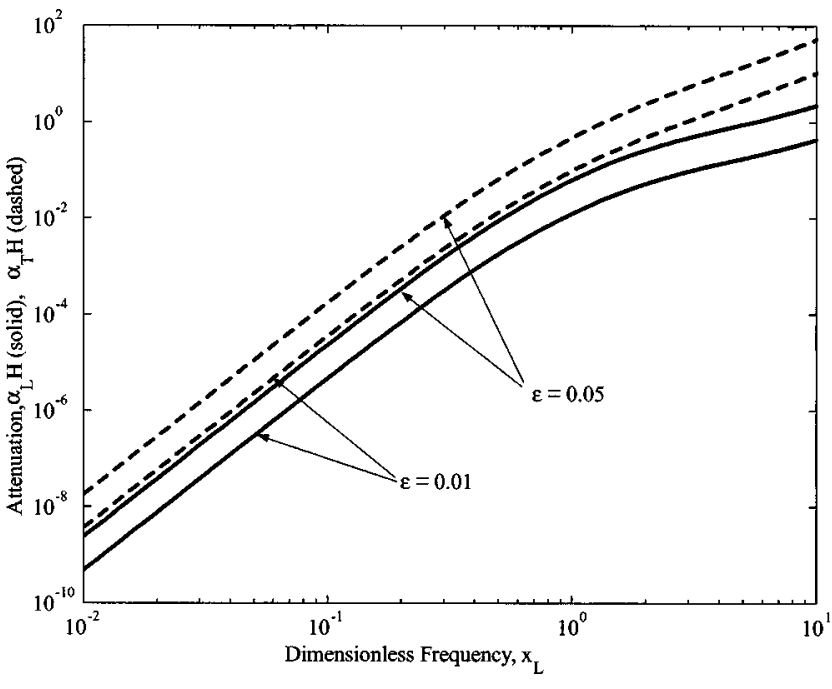

FIG. 2. Dimensionless longitudinal and transverse attenuations, $\alpha_{L} H$ (solid) and $\alpha_{T} H$ (dashed), as a function of dimensionless frequency, $x_{L}$, for damage factor $\varepsilon=0.01$ and $\varepsilon=0.05$.

with the square of frequency. However, it should be noted that the results at higher frequencies are less accurate than those at lower frequencies. This inaccuracy is the result of the truncation of the expansion in Eqs. (20). The longitudinal attenuation is smaller than the transverse attenuation in part due to the wavelengths of the respective waves. However, when the attenuations are plotted in terms of their respective dimensionless frequency, the transverse attenuations remained larger than the longitudinal. Thus, the higher transverse attenuation is a combination of effects of wavelength and interaction with the cracks. As shown in Fig. 2, the ratio of the longitudinal and transverse attenuations is a constant at low frequencies, but changes at higher frequencies. Figure 3 is a plot of the wave speed ratio $B^{e}=\bar{C}_{T} / \bar{C}_{L}$ and $C$ $=\left(\bar{C}_{T} / C_{T}\right) /\left(\bar{C}_{L} / C_{L}\right)$, as a function of damage factor $\varepsilon$. As has been observed experimentally, the wave speed changes much less than the attenuation for a given damage level. ${ }^{30}$ Thus, the result shown in Fig. 3 is not unexpected.

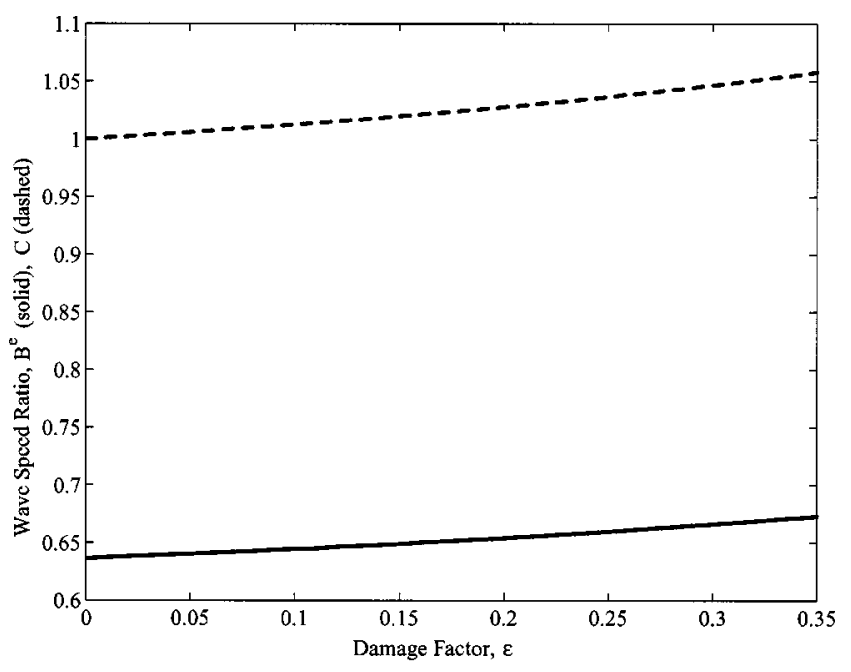

FIG. 3. Wave speed ratio $B^{e}=\bar{C}_{T} / \bar{C}_{L}$ and $C=\left(\bar{C}_{T} / C_{T}\right) /\left(\bar{C}_{L} / C_{L}\right)$, as a function of damage factor $\varepsilon$. 


\section{DISCUSSION}

The propagation and scattering of elastic waves in a homogeneous, isotropic medium with damage from microcracking has been investigated. A generalized tensor-based approach was used such that the results are coordinate free. The effective compliance or stiffness in terms of the damage parameter was discussed. Initially, effective compliance due to a single penny-shaped crack embedded in an infinite elastic solids was examined. The effective properties of a homogenous, isotropic solid in which a large number of microcracks is embedded were then determined by superposition. The modulus fluctuations were then derived relative to these average moduli. The ensemble average covariance of the modulus fluctuations was then derived for randomly oriented cracks. The expressions for the longitudinal and transverse attenuations were derived by considering the Dyson equation, which governs the mean elastodynamic response of the medium. The Dyson equation was solved within the limits of first-order smoothing approximation (FOSA). The final forms of the attenuations have a linear dependency on the damage parameter, which is expected to be valid for low crack densities. The use of the tensor-based approach presented here for studying elastic wave scattering in media with microcracks allowed the attenuation expressions to be reduced to simple forms. These results should be very useful for nondestructive testing and materials characterization research. In particular, the study of wave interactions with aligned cracks, whose evolution is modeled using a damage tensor, will be much more direct. This topic will be examined in the future. The general formulation also is convenient for considering other problems such as studies of backscatter and multiple scattering phenomena.

\section{ACKNOWLEDGMENTS}

The support of the National Science Foundation (Grants No. CMS-9978707 and INT-0089548) and the National Bridge Research Organization (NaBRO) is gratefully acknowledged by authors.

${ }^{1}$ J. Achenbach, "Quantitative Nondestructive Evaluation," Int. J. Solids Struct. 37, 13-27 (2000).

${ }^{2}$ C. Zhang and J. Achenbach, "Effective Wave Velocity and Attenuation in a Material with Distributed Penny-Shaped Cracks," Int. J. Solids Struct. 27, 751-767 (1991)

${ }^{3}$ A. K. Mal, "Interaction of Elastic Waves with a Penny-Shaped Crack," Int. J. Eng. Sci. 8, 381-388 (1968).

${ }^{4}$ A. K. Mal, "A Note on the Low Frequency Diffraction of Elastic Waves by a Griffith Crack,” Int. J. Eng. Sci. 10, 609-612 (1972).

${ }^{5}$ M. Piau, "Attenuation of a Plane Compressional Wave by a Random Distribution of Thin Circular Cracks," Int. J. Eng. Sci. 17, 151-167 (1979).
${ }^{6}$ A. Chatterjee, A. K. Mal, L. Knopoff, and J. Hudson, "Attenuation of Elastic Waves in a Cracked, Fluid-Saturated Solid," Math. Proc. Cambridge Philos. Soc. 88, 547-561 (1980).

${ }^{7}$ C. Zhang and D. Gross, "Wave Attenuation and Dispersion in Randomly Cracked Solids-II. Penny-Shaped Cracks," Int. J. Eng. Sci. 31, 859-872 (1993).

${ }^{8}$ V. P. Smyshlyaev and J. R. Willis, "Linear and Nonlinear Scattering of Elastic Waves by Microcracks," J. Mech. Phys. Solids 42, 585-610 (1994).

${ }^{9}$ S. Hirsekorn, "The scattering of ultrasonic waves by polycrystals," J. Acoust. Soc. Am. 72, 1021-1031 (1982).

${ }^{10} \mathrm{~S}$. Hirsekorn, "The scattering of ultrasonic waves by polycrystals. II. Shear waves," J. Acoust. Soc. Am. 73, 1160-1163 (1983).

${ }^{11}$ F. E. Stanke and G. S. Kino, "A unified theory for elastic wave propagation in polycrystalline materials," J. Acoust. Soc. Am. 75, 665-681 (1984).

${ }^{12}$ R. L. Weaver, "Diffusion of Ultrasound in Polycrystals," J. Mech. Phys. Solids 38, 55-86 (1990).

${ }^{13} \mathrm{~J}$. A. Turner, "Elastic wave propagation and scattering in heterogeneous, anisotropic media: Textured polycrystalline materials," J. Acoust. Soc. Am. 106, 541-552 (1999).

${ }^{14}$ J. Lemaitre, A Course on Damage Mechanics (Springer-Verlag, Berlin, 1992).

${ }^{15}$ D. Krajcinovic, Damage Mechanics (North-Holland, Amsterdam, 1996).

${ }^{16} \mathrm{~S}$. Nemat-Nasser and M. Hori, Micromechanics: Overall Properties of Heterogeneous Materials (North-Holland, Amsterdam, 1993).

${ }^{17}$ M. Kachanov, "Elastic Solids with Many Cracks and Related Problems," Adv. Appl. Mech. 29, 259-445 (1993).

${ }^{18}$ Z. Hashin and S. Shtrikman, "A Variational Approach to the Theory of the Elastic Behavior of the Multiphase Materials," J. Mech. Phys. Solids 11, 127-140 (1963)

${ }^{19}$ L. V. Gibiansky and S. Torquato, "Link Between the Conductivity and Elastic Moduli of Composite Materials," Phys. Rev. Lett. 71, 2927-2930 (1993).

${ }^{20}$ U. Frisch, "Wave Propagation in Random Media," in Probabilistic Methods in Applied Mathematics, edited by A. T. Barucha-Reid (Academic, New York, 1968), Vol. 1, pp. 75-198.

${ }^{21}$ F. C. Karal and J. B. Keller, "Elastic, electromagnetic, and other waves in a random medium," J. Math. Phys. 5, 537-547 (1964).

${ }^{22}$ V. Lubarda and D. Krajcinovic, "Tensorial Representations of the Effective Elastic Properties of the Damaged Material,” Int. J. Damage Mech. 3, 38-56 (1994).

${ }^{23}$ I. Kunin, Elastic Media with Microstructure II (Springer-Verlag, Berlin, 1983).

${ }^{24}$ J. Walsh, "The Effect of Cracks on Compressibility of Rocks," J. Geophys. Res. 70, 381-389 (1965).

${ }^{25}$ B. Budiansky and R. J. O'Connell, "Elastic Moduli of a Cracked Solid," Int. J. Solids Struct. 12, 81-97 (1976).

${ }^{26}$ M. Kachanov, I. Tsukrov, and B. Shafiro, "Effective Moduli of Solids with Cavities of Various Shapes," Appl. Mech. Rev. 47, 151-174 (1994).

${ }^{27}$ R. W. Zimmerman, "The Effect of Microcracks on the Elastic Moduli of Brittle Materials," J. Mater. Sci. Lett. 4, 1457-1460 (1985).

${ }^{28}$ K. Z. Markov and J. R. Willis, "On the Two-Point Correlation Function for Dispersions of Nonoverlapping Spheres," Math. Models Methods Appl. Sci. 8, 359-377 (1998).

${ }^{29}$ F. E. Stanke, "Spatial autocorrelation functions for calculations of effective propagation constants in polycrystalline materials," J. Acoust. Soc. Am. 80, 1479-1485 (1986).

${ }^{30}$ S. F. Selleck, E. N. Landis, M. L. Peterson, S. P. Shah, and J. D. Achenbach, "Ultrasonic Investigation of Concrete with Distributed Damage," ACI Mater. J. 95, 27-36 (1998). 